\title{
Prosthetic Reconstruction of Auricular Defects with an Adhesive-Retained Epithesis: A Clinical Report
}

Murat Yenisey, Necati Kaleli* and Göknil Ergün Kunt

Department of Prosthodontics, Faculty of Dentistry, University of Ondokuz Mayis

\begin{abstract}
Craniofacial implants have been widely used for the long-term retention of ear epithesis. However, previous surgical failures and patient preferences may contraindicate the implantation in some cases. This clinical report presents the prosthetic reconstruction of auricular defects without implant anchorage. An adhesive-retained ear epithesis was designed for two patients. The margins of epitheses were sealed by using tissue adhesives; this along with the mechanical support of tissue undercuts promoted the retention of epitheses. Patients were clinically evaluated during follow-ups at 1, 3, and 6 months; they had no complaints and were satisfied with their appearance.
\end{abstract}

Keywords: Auricular defects; Auricular epithesis; Tissue; Adhesives

\section{Introduction}

An auricular defect may result from congenital or acquired anomalies such as trauma from burns, accidents, and surgical removal of tumoral lesions [1-3]. These patients usually suffer from functional as well as psychological problems, which affect their social life because of esthetic concerns [4-6]. Therefore, reconstruction of these defects has a great importance to ensure a better quality of life. Auricular defects can be reconstructed surgically (autogenous reconstruction) or prosthetically, but the choice of the treatment depends on factors such as location, size and type of the defect, systemic or local health status, and preferences of the patient [7-9]. Although autogenous reconstruction has remarkable advantages, prosthetic reconstruction minimizes the potential risks of surgery and can provide high quality aesthetic results [9-11].

Epithesis retention is one of the key factors determining the success of prosthetic reconstruction and is also important for patient comfort. Many clinical studies have reported that the use of osseointegrated implants provides a satisfactory retention and improves the acceptance of auricular epithesis [12-16]. Further, craniofacial implants placed in the auricular region have been reported to have excellent survival rates [17-19]. Despite the high success rate, failures in previous surgical attempts and preferences of the patient may restrict the implantation. Tissue adhesives and anatomical retention factors are at the forefront in such cases. This clinical report presents the prosthetic rehabilitation of two patients who had undergone several auricular reconstructive surgeries without the use of craniofacial implants.

\section{Case Presentation}

Two male patients, both approximately 20 years of age, had been referred to the Department of Prosthodontics (Ondokuz Mayis University, Faculty of Dentistry), complaining of ear malformation. The first patient had undergone tumoral resection surgery of the right ear and several subsequent reconstructive surgeries (Figure 1A). The second patient suffered from a congenital absence of the left ear, with a history of an unsuccessful, autogenous reconstruction (Figure 1B). Both patients were dissatisfied with the result of reconstructive surgeries. An adhesive-retained ear epithesis was indicated for both patients, based on clinical and radiographic examination and as per their choice.

Impressions of healthy ears and auricular defects were taken with hydrocolloid impression material (Cavex Impressional, Cavex). Cast models were poured by using type 3 dental stone (Giludur, BK Giulini). Thereafter, wax patterns of epitheses (Figure 2) were prepared by using dental wax (Cavex Set Up Wax, Cavex) based on cast models and photographs of healthy ears. After the trial of wax patterns on patients in terms of marginal fit and anatomical localization, cast models were molded (Figure 3). The molds were then placed in $100{ }^{\circ} \mathrm{C}$ boiling water for $20 \mathrm{~min}$ to remove all the wax material (Figure 4). An isolant liquid (C.M.S., Dentsply) was applied to all pieces before condensation of silicone material. A heat temperature maxillofacial silicone (Cosmesil Series M511, Principality Medical) was used to fabricate epitheses. The catalyst and putty parts were mixed according to the manufacturer's instructions. Color pigments and fibers (Cosmesil, Principality Medical) were primarily used in intrinsic coloration of silicone material. At first, small pieces of silicone material in different skin shades were condensed to external margins in a step-by-step manner to reflect all surface colors (Figure 5). Subsequently, a principal skin shade was prepared and applied to all parts of molds. Coloration process was performed by using photographs of healthy ears as well as a skin shade to match computer and daylight visualization. After condensing silicone material, molds were placed in $100^{\circ} \mathrm{C}$ boiling water for 1 hour. After the polymerization process and finishing procedures, extrinsic coloring agents (Cosmesil, Principality Medical) were applied to external surfaces (Figure 6). Final adjustments were made on patients. Margins of epitheses were sealed by using a tissue adhesive (Pro Bond, Principality Medical) and delivered to patients (Figure 7). Mechanical support of tissue undercuts also promoted the retention of epitheses.

\section{Discussion}

Auricular defects can be reconstructed surgically (autogenous reconstruction) or prosthetically, and each method has some advantages and disadvantages. A successful autogenous reconstruction

*Corresponding author: Necati Kaleli, Researcher, Department of Prosthodontics, Faculty of Dentistry, University of Ondokuz Mayis, Turkey, Tel: +9036231219192783; E-mail: necati_kaleli@hotmail.com

Received: November 20, 2016; Accepted: November 23, 2016; Published: November 30, 2016

Citation: Yenisey M, Kaleli N, Ergün Kunt G (2016) Prosthetic Reconstruction of Auricular Defects with an Adhesive-Retained Epithesis: A Clinical Report. Reconstructive Surgery and Anaplastology 5: 163. doi: 10.4172/21611173.1000163

Copyright: ( 2016 Yenisey M, et al. This is an open-access article distributed under the terms of the Creative Commons Attribution License, which permits unrestricted use, distribution, and reproduction in any medium, provided the original author and source are credited. 
Citation: Yenisey M, Kaleli N, Ergün Kunt G (2016) Prosthetic Reconstruction of Auricular Defects with an Adhesive-Retained Epithesis: A Clinical Report. Reconstructive Surgery and Anaplastology 5: 163. doi: 10.4172/2161-1173.1000163

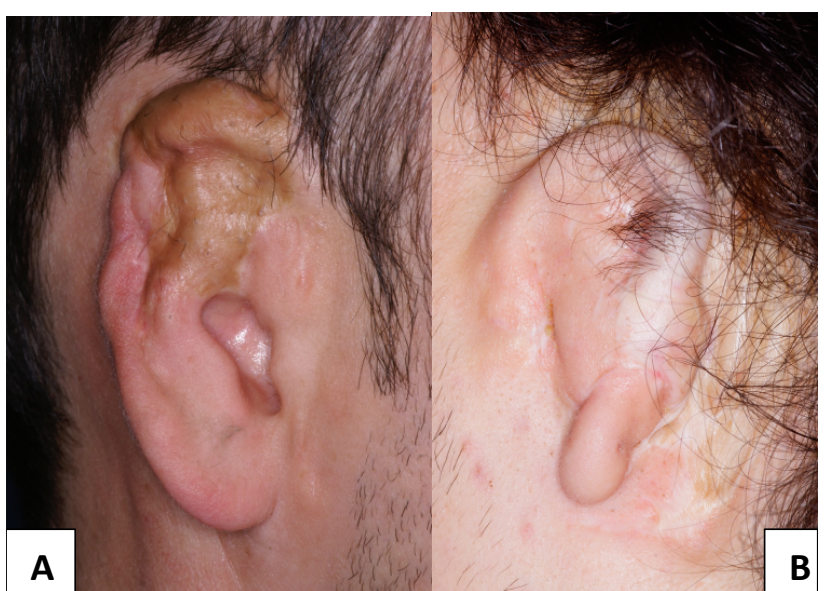

Figure 1: Auricular defects of patients. A) First patient. B) Second patient.

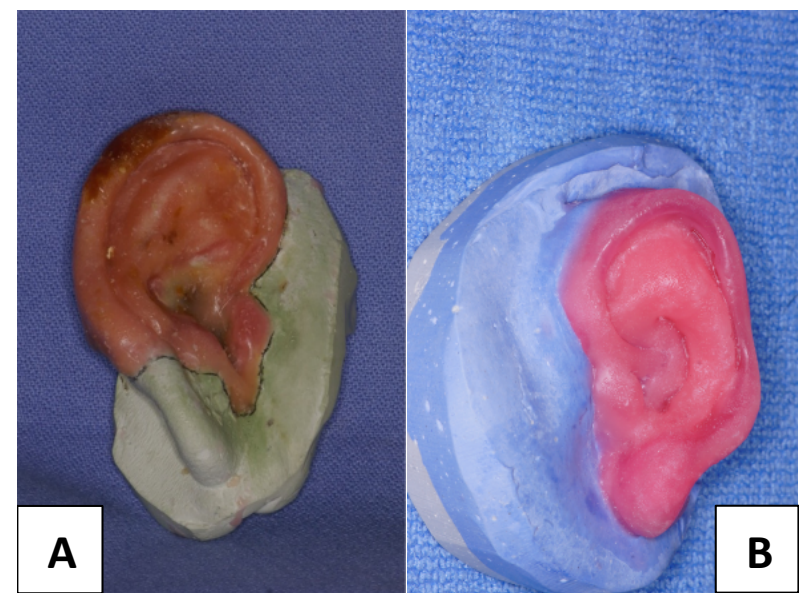

Figure 2: Wax patterns of epitheses. A) First patient. B) Second patient.

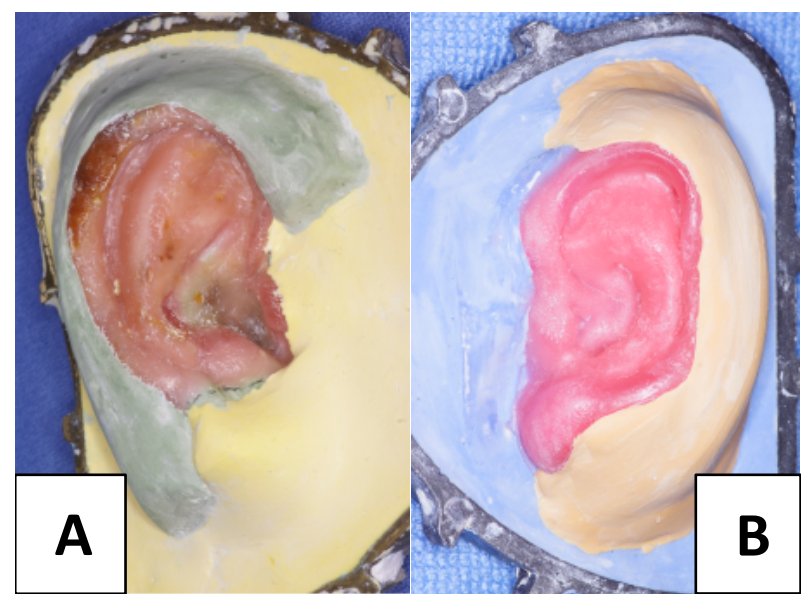

Figure 3: Molded cast models. A) First patient. B) Second patient.

requires no further treatment, maintains the natural texture of skin, and helps patients immediately improve their self-esteem. On the other hand, prosthetic reconstruction requires periodic replacement of the

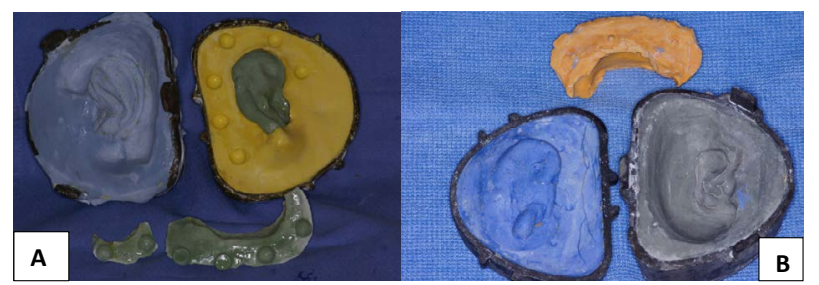

Figure 4: Wax removal. A) First patient. B) Second patient.

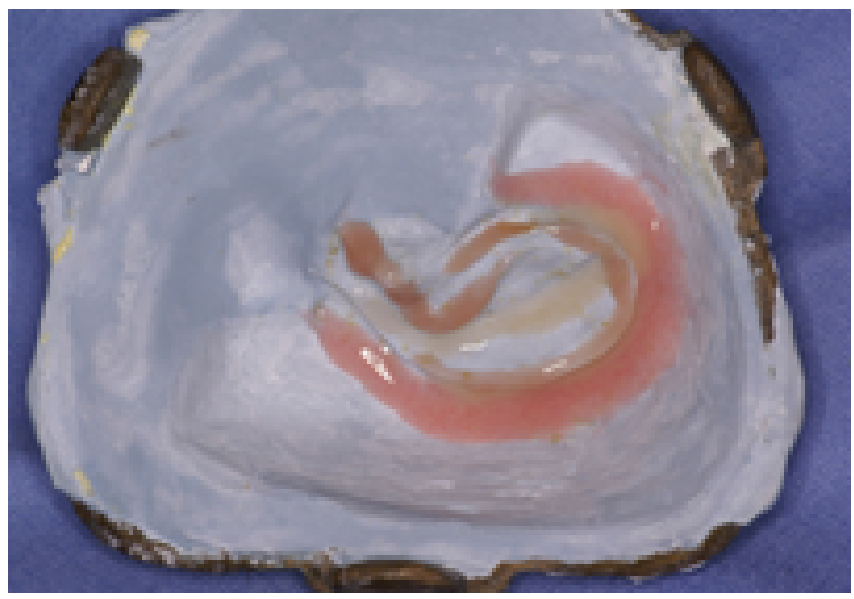

Figure 5: Application of first silicon layer.

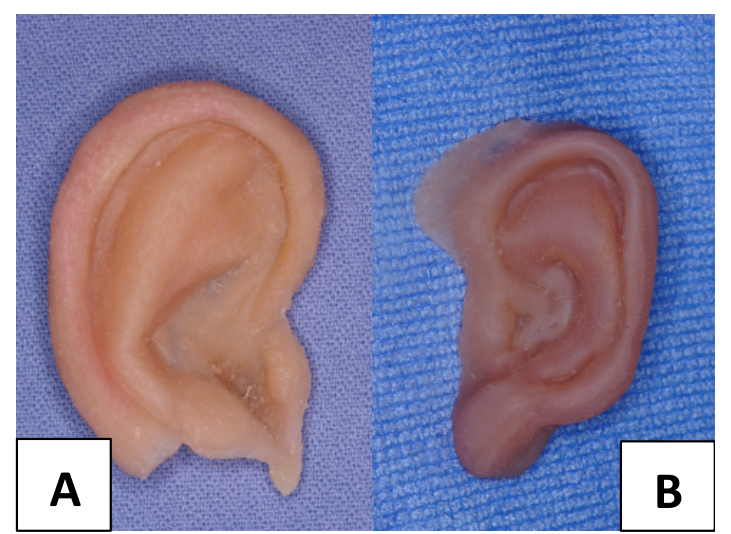

Figure 6: Finished epitheses. A) First patient. B) Second patient.

epithesis for a lifetime, and it is recommended that patients remove their epithesis every night. This situation makes the acceptance of epithesis difficult as it regularly reminds the patient of their deformity [11]. However, autogenous reconstruction also has considerable disadvantages including longer surgical procedures, postoperative pain and scarring at several operative sites, and the possibility of the final reconstructed ear not being similar to the healthy opposite ear [9]. An experienced anaplastologist can create a high quality epithesis that is quite identical to a healthy one. Also, autogenous reconstruction cannot be indicated for every patient. Cancer patients who have undergone resection surgery are irradiated frequently, which disrupts local tissue health [10]. Thorne et al. [11] summarized potential indications to facilitate decision-making for using prosthetic reconstruction: previous failed autogenous reconstructions, congenital severe soft tissue or 


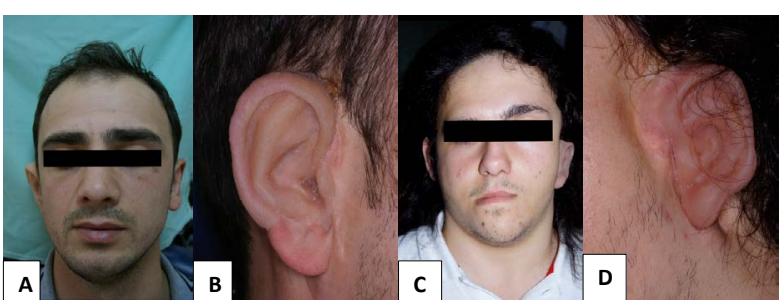

Figure 7: Delivered epitheses. A) Frontal view of first patient. B) Lateral view of first patient. C) Frontal view of second patient. D) Lateral view of second patient.

skeletal hypoplasia, low or unfavorable hairline, and posttraumatic auricular defects. If systemic and local health conditions are unsuitable for autogenous reconstruction, prosthetic rehabilitation is a valuable alternative.

Prosthetic reconstruction of auricular defects presents a great challenge to anaplastologists due to the absence of hard or soft tissue undercuts [12]. Prior to the introduction of craniofacial implants, auricular epitheses were retained by using mechanical tools (spectacle frames, headbands, and steel springs), skin adhesives, or mechanical support of anatomical undercuts (when available) [1,4,13,1618]. However, skin adhesives may lead to adverse tissue reactions, discoloration and deformation at edges of the epithesis, loss of adhesion because of perspiration, and difficulties in maintaining the position of the epithesis $[5,12,16-18]$. On the other hand, craniofacial implants offer higher retention, allow easier positioning of the epithesis, and eliminate the use of adhesives, thus extending the epithesis survival $[14,20]$. Moreover, craniofacial implants placed in the auricular region have remarkable survival rates [17-19] even in irradiated patients. A statistically significant relationship between history of radiation therapy and survival of implants has not been identified [18]. Also, the mastoid process provides excellent bone quality and volume for osseointegration and promotes the survival of auricular epithesis [13,18]. Despite overwhelming advantages, an implant-retained epithesis is not a cost-effective treatment for economically disadvantaged patients [7]. Besides, as previously mentioned, reconstructive surgeries may contraindicate the implantation procedure. If craniofacial implants are used in such cases, auricular epitheses should be thicker than usual to mask both prosthetic attachments and surgical failure of autogenous graft, and this may cause an asymmetrical appearance. In the present cases, an adhesive-retained epithesis was indicated for both patients owing to previous failed reconstructive surgeries and their reluctance to undergo another surgery.

\section{Conclusion}

Craniofacial implants offer higher retention rates than skin adhesives, improve the acceptance of prosthesis, and provide a better quality of life. However, prior surgical operations and patient preferences may contraindicate the use of craniofacial implants. Skin adhesives and mechanical support of anatomical undercuts may be the only option in such situations.

\section{References}

1. Özçelik TB, Tanner PB (2010) A surgical guide for craniofacial implant placement for an implant-retained auricular prosthesis. J Prosthet Dent 103 253-255.

2. Gupta S, Gupta S, Pandey A (2016) Auricular defects: Autogeneous vs prosthetic reconstruction. Int J Med Res Rev 4: 387-391.

3. Pellicano JM, Oliveria CACP, Bahmad F (2015) Ear rehabilitation- Is it time for the osseointegrated prostheses? Systematic review. Anaplastology 4: 148.

4. Ariani N, Visser A, Van Oort RP, Kusdhany L, Rahardjo TB, et al. (2013) Current state of craniofacial prosthetic rehabilitation. Int J Prosthodont 26: 57-67.

5. Wondergem M, Lieben G, Bouman S, Brekel MWMVD, Lohuis PJFM (2016) Patients' satisfaction with facial prostheses. Br J Oral Maxillofac Surg 54: 394 399.

6. Chang TL, Garrett N, Roumanas E, Beumer J (2005) Treatment satisfaction with facial prostheses. J Prosthet Dent 94: 275-280.

7. Rani G, Gambhir A (2015) Reconstruction of auricular defect with adhesiveretained silicon prosthesis-A case report. Int J Dent Oral Health.

8. Ciorba A, Martini A (2005) Tissue engineering and cartilage regeneration for auricular reconstruction. Int J Pediatr Otorhinolaryngol 70: 1507-1515.

9. Wilkes GH, Wolfaardt JF (1994) Osseointegrated alloplastic versus autogenous ear reconstruction: Criteria for treatment selection. Plast Reconstr Surg 93 967-979.

10. Wolfaardt J, Gehl G, Farmand M, Wilkes G (2003) Indications and methods of care for aspects of extraoral osseointegration. Int J Oral Maxillofac Surg 32 124-131.

11. Thorne $\mathrm{CH}$, Brecht LE, Bradley JP, Levine JP, Hammerschlag $\mathrm{P}$, et al (2001) Auricular reconstruction: Indications for autogeneous and prosthetic techniques. Plast Reconstr Surg 107: 1241-1252.

12. Nishimura RD, Roumanas E, Sugai T, Moy PK (1995) Auricular prostheses and osseointegrated implants: UCLA experience. J Prosthet Dent 73: 553-558.

13. Arora V, Sahoo NK, Gopi A, Saini DK (2016) Implant-retained auricular prostheses: A clinical challenge. Int J Oral Maxillofac Surg 45: 631-635.

14. Shrestha B, Fatmasari F, Thaworanunta S, Srithavaj T (2015) Prosthodontic rehabilitation of congenital auricular defect: A clinical report. Cleft Palate Craniofac J 52: 229-233.

15. Thomas KF (1995) Freestanding magnetic retention for extraoral prosthesis with osseointegrated implants. J Prosthet Dent 73: 162-165.

16. Wright RF, Zemnick C, Wazen JJ, Asher E (2008) Osseointegrated implants and auricular defects: A case series study. J Prosthodont 17: 468-475.

17. Chrcanovic BR, Nilsson J, Thor A (2016) Survival and complications of implants to support craniofacial prosthesis: A systematic review. J Craniomaxillofac Surg 44: $1536-1552$.

18. Curi MM, Oliveira MF, Molina G, Cardoso CL, De Groot Oliveira L, et al. (2012) Extraoral implants in the rehabilitation of craniofacial defects: Implant and prosthesis survival rates and peri-implant soft tissue evaluation. $\mathrm{J}$ Oral Maxillofac Surg 70: 1551-1557.

19. Granström G (2007) Craniofacial osseointegration. Oral Dis 13: 261-269.

20. Reisberg DJ, Habakuk SW (1995) Hygiene procedures for implant-retained facial prostheses. J Prosthet Dent 74: 499-502 